\title{
An Overview of Dual Targeting Nanostructured Lipid Carriers for the Treatment of Ovarian Cancer
}

\author{
Praharsh Kumar Mandadhi Rajendra1, Natarajan Jawahar ${ }^{1, *}$, Rajeshkumar Raman ${ }^{2}$, Hagalavadi \\ Nanjappa Shivakumar ${ }^{3}$, Anjali Puthusserikkunnu Balan'
}

${ }^{1}$ Department of Pharmaceutics, JSS College of Pharmacy, JSS Academy of Higher Education \& Research, Ooty, Nilgiris, Tamil Nadu, INDIA.

2Department of Pharmaceutical Biotechnology, JSS College of Pharmacy, JSS Academy of Higher Education \& Research, Ooty, Nilgiris, Tamil Nadu, INDIA.

${ }^{3}$ Department of Pharmaceutics, KLE College of Pharmacy, Bengaluru, Karnataka, INDIA

\begin{abstract}
Purpose: To summarize main findings from research on oral delivery of Nanostructured lipid carriers targeting ovarian cancer. Methods: A narrative review of all the relevant papers known to author was conducted. Results: Ovarian cancer is one of the most common gynaecologic cancer, the frontline treatments for which are surgical approach followed by radiotherapy or chemotherapy. Chemotherapy has its own limitations; consequently, there is a need to develop a targeted drug delivery system with high efficacy. Preclinical evidence suggests that combination therapies are likely to be effective in ovarian cancer. However, from clinical reports, it is evident that chemotherapeutic drugs have lower therapeutic value for the treatment of ovarian cancer. We propose that these limitations can be overcome by a novel formulation consisting of nanostructured lipid carriers. Recent pharmacological research has successfully potentiated the effects of combination therapy in acute animal preparations by inhibiting proteins that are involved in different physiological pathways of cancer cells. Conclusion: We have reviewed the scope of nanostructured lipid carriers concerning chemotherapy of ovarian cancer. The advantages of oral administration of these lipid carriers are discussed. Also, the various nano-lipid formulations and combination therapies reported along with their therapeutic outcomes have been reviewed. In light of this, nanostructured lipid carriers containing two different active ingredients for oral administration could be a potential approach for the effective treatment of ovarian cancer.
\end{abstract}

Key words: Ovarian cancer, Nanostructured lipid carriers, Dual targeting,

Chemotherapy, Oral delivery.

\section{INTRODUCTION}

One of the lethal and second most common gynecologic malignancies in the world is ovarian cancer (OC) which has a high mortality rate. Around $70 \%$ of cases are diagnosed at an advanced stage. This is due to the secret growth of the tumour, delayed onset of symptoms, and lack of proper screening., ${ }^{1,2}$ OC treatment employs invasive surgery for the removal of affected ovaries, fallopian tubes, and cervix. This is followed by radiotherapy or chemotherapy, based on the stage of OC identified. Intravenous cisplatin and paclitaxel (PTX) are conventional drugs used for the treatment of OC. However, conventional therapy has its own limitations and drawbacks that include subsequent disease relapse, development of drug resistance, and toxicities. Several side effects including nausea, alopecia, and declination in plasma counts are associated with chemotherapy treatment when administered to patients having OC. The combination of PTX and Methotrexate results in decreased bioavailability, increased toxicity, and drug incompatibility thereby reducing cancer targeting. To surpass treatment drawbacks of conventional therapeutics,
Submission Date: 01-07-2020; Revision Date: 02-12-2020; Accepted Date: 01-03-2021

DOI: 10.5530/ijper.55.2.70 Correspondence: Dr. Natarajan Jawahar Department of Pharmaceutics, JSS College of Pharmacy, Ooty, Tamil Nadu, INDIA.

Phone no: +91 9791439545 Email id: jawahar.n@jssuni. edu.in

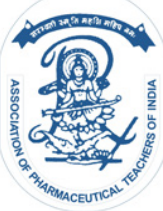

www.ijper.org 
various targeting drug delivery systems are developed. These delivery systems direct anticancer therapeutics to tumour sites specifically. Also, advanced polymeric nanotechnology provides better alternatives for treating OC, thereby minimizing systemic toxicities linked with the administration of the chemotherapeutic drug. ${ }^{3}$

Also, a combination of chemotherapeutics can be used to improve anticancer efficacy against OC. This depends on the theory that one drug-resistant tumour cells will be sensitive to another drug with a different mechanism of action. Research has witnessed exceptional growth over the past two decades in using nanotechnology for therapeutic applications, particularly in oncology. Nanotechnology increases drug-tumour accumulation and reduces off-target toxicity, consequently achieving higher treatment efficiency. ${ }^{4}$ Nanostructured lipid carriers (NLCs) have shown to be a reliable platform for the delivery of anticancer drugs in the treatment of OC. NLCs have distinct advantages that make them be considered as promising nano-systems. Oral administration of these NLCs can be regarded as a better approach for the treatment of chronic diseases like diabetes, hypertension, and cancer. All these advantages are discussed in the following sections. Combination therapy for dual targeting can be adopted with NLCs via oral administration for effective treatment of OC. This reduces adverse effects, sustains the release of drugs thereby enhancing the anticancer efficacy with longer shelf storage stability. Systemic toxicity can be decreased and since these are administered orally, patient compliance and ease of administration are attained. Dual targeting can be achieved by loading two drugs with a different mechanism of action like Paclitaxel and Hydroxyurea into an NLC. Here, Paclitaxel acts on TUBB that is involved in cell division, and Hydroxyurea acts on RRM2 which is responsible for purine, pyrimidine, and

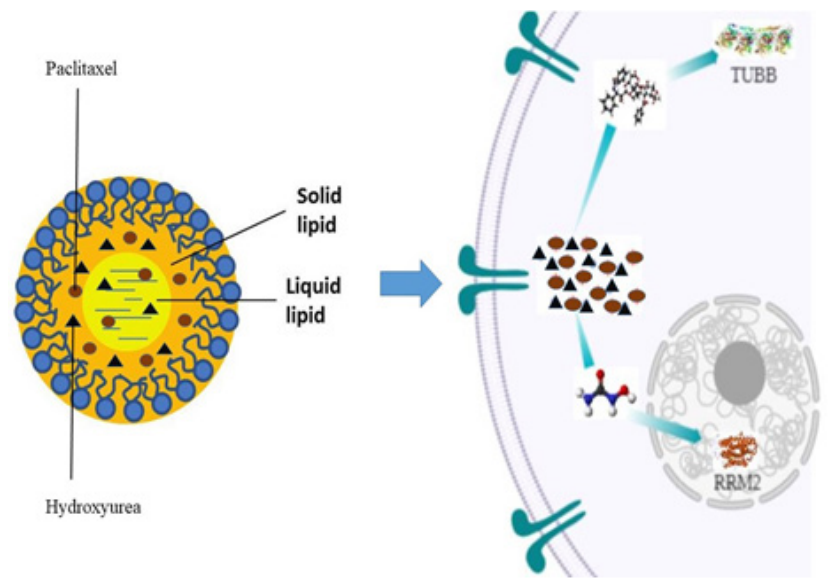

Figure 1: NLC loaded with two drugs targeting cancer cells. Paclitaxel acts on TUBB and Hydroxyurea act on RRM2. glutathione metabolism. Figure 1 describes the NLC loaded with these two drugs.

\section{Scope of NLCs in chemotherapy}

It is evident from recent studies that NLCs enhance efficacy, stability, and reduces the side effects of cytotoxic drugs. Various nano-systems carrying anticancer drugs have been developed like albumin-paclitaxel nanoparticles for breast cancer, which was approved for chemotherapy in early 2005. For lung carcinoma cells etoposide NLCs were found to be cytotoxic. Topotecan NLCs were developed which were stable and prolonged the release of drugs for treating ovarian and smallcell lung cancer. These NLCs have various advantages such as high drug loading efficacy, prolonged-release characteristics, improved stability, increased cytotoxicity when incorporated with anticancer drugs. Some potential problems associated with SLN that include drug leakage and decreased loading capacity can be avoided by these NLCs. They prolong the exposure of tumour cells to chemotherapeutic agents and enhance permeability and retention, which thereby increases therapeutic index. These NLCs avoid some potential problems associated with SLN, such as drug leakage during storage and decreased loading capacity. They act by prolonging the exposure of tumour cells to antitumour drugs and enhancing permeability and retention effect to further increase the therapeutic effect. ${ }^{5,6}$

NLCs were developed by Muller $e t$ al. They used mixtures of solid lipids and liquid lipids which at both room and body temperature form an amorphous solid matrix. The fundamental step is the incorporation of liquid lipids in the matrix that significantly enhances formulation properties when compared to SLNs. Liquid lipids create an amorphous lattice with considerable imperfections in the crystalline solid matrix by which higher drug loading can be attained. For various therapeutic and cosmetic applications, NLCs were studied as delivery platforms. They have significantly higher encapsulation efficiency, bioavailability, controlled release, biocompatibility, and safety profiles. ${ }^{7-9}$

A tumour is affiliated with defective and leaky vascular. This is due to a poorly regulated tumour angiogenesis environment. Within the tumour, the interstitial fluid is insufficiently drained by a poorly formed lymphatic system. As an outcome, a submicron-sized particulate issue may preferentially extravasate into the tumour and be kept there. This is often mentioned as the "enhanced permeability and keeping" (EPR) effect. ${ }^{10}$ The EPR effect can be fruitful by the proper design of nanoparticles like NLCs for passive tumour targeting. This partially solves the poor specificity problems. 
Also, surface engineering of these NLCs can alter their biodistribution properties. Surface engineering involves the modification of external physicochemical properties of NLCs, which directs them to the selected tissue. This enhances the target specificity of the formulation and reduces systemic toxicity. ${ }^{8,10}$ Thus, it is convincible that anticancer drugs administered to treat OC can be loaded into NLCs so that tumour targeting is achieved and thereby reducing systemic adverse effects.

\section{NLCs targeting ovarian cancer}

Various NLCs loaded with anticancer drugs for the treatment of OC have been reported. Few of them have been reviewed in the following.

Liping Wang et al. reviewed that ovarian tumours express CD44 family of cell surface proteoglycans whose natural ligand is hyaluronic acid (HA). They aimed to develop HA-based paclitaxel (PTX) loaded NLCs to improve OC therapeutic outcomes. They prepare cationic PTX-NLCs. By electrostatic absorption technique, they coated HA to these NLCs to obtain HA-PTX-NLCs. In vitro and in vivo studies revealed that there was an enhanced reduction in tumour growth when administered with these NLCs compared to PTX injection. They demonstrated that significant results were due to the usage of novel HA-PTX-NLCs. ${ }^{11}$

Pooja Mittal et al. focused on the formulation and characterization of genistein NLCs for sustained drug release for OC treatment. Genistein is a natural flavonoid that has anticancer activity. Its drawbacks include poor solubility and low oral bioavailability. To overcome these they developed genistein NLCs by solvent emulsification and evaporation technique. Pharmacokinetic and biodistribution studies of these NLCs revealed that plasma drug concentration for a longer time period and improved drug distribution in OC tissues was achieved. They concluded that genistein NLCs seem to be an alternative for higher entrapment and excellent stability. ${ }^{12}$

Maria Luisa Bondi et al. studied curcumin that is a natural molecule possessing an anti-cancer effect. Its clinical use has been limited as its bioavailability is poor. They believed that nanocarrier drug delivery systems would overcome the limitations of curcumin. They aimed to increase the bioavailability of curcumin by loading into NLCs. They prepared compritol NLCs considering its slower drug release characteristics. Their in vitro results showed that these NLCs had better anticancer efficacy than free curcumin. This shows that NLCs loaded with curcumin have potential drug delivery profiles in the treatment of $\mathrm{OC} .^{13}$
Ki Hyun Bang et al. reviewed that NLCs are emerging tools that improve tumour treatment outcomes. They designed PTX NLCs with platelet membrane proteins as these proteins are involved with the angiogenesis and interaction of circulating tumour cells. They coated PTX NLCs with platelet membrane proteins isolated from blood by the gravity-gradient method. Transmission electron microscopy, western blot, and ELISA confirmed this coating process. In vitro cell studies displayed that these NLCs had improved antitumor effect towards SK-OV-3 cells. They summarized that these PTX NLCs had an affinity and targeting ability for OC cells. ${ }^{14}$

\section{NLCs for oral delivery}

For treating various deadly diseases, the oral route of administration of drugs is a valuable option. It has several advantages which makes it to be the most commonly accepted route of administration. These advantages include patient compliance, cost-effectiveness, and ease of administration. While administering chronic agents such as antidiabetic, antihypertensive, and anticancer agents this route is highly preferred. ${ }^{15}$ More than $40 \%$ of drugs emerging from the drug discovery process are unfortunately not suitable for oral delivery because of their hydrophobicity and poor oral bioavailability. First pass metabolism, drug expulsion via P-glycoprotein, and food effects are other barriers that are encountered with this route. This shows that there is a high necessity for the development of oral drug delivery systems which overcome the aforementioned factors for desired therapeutic activities. ${ }^{15,16}$

Various attempts and approaches are being designed in research to overcome these challenges and bioavailability enhancement with respect to oral drug delivery platforms. Prodrug strategies, slat formation, nano-encapsulation of drugs via polymeric micelles, emulsions, liposomes, etc. are a few methods adopted for this purpose. ${ }^{17}$ Over the past few decades, lipid drug delivery systems showed better effects on drug absorption. Reports suggest that liposomes, micelles, emulsion, and other conventional lipid systems are susceptible to degradation during storage and in the acidic environment of the stomach. NLCs have been consistently reported for increased entrapment efficiency. ${ }^{17}$ This is attributed to the structural parity of two lipids in NLCs resulting in structural imperfections which while solidification gives more space for drugs. ${ }^{18}$ The drugs also possess more solubility in liquid lipids than solid lipids. These NLCs have longer storage stability. All these properties make NLCs to be considered as advanced carriers when compared to conventional lipid-based drug delivery systems. ${ }^{19}$ NLCs can be incorporated with lipophilic and 
hydrophilic drugs. They also provide sustained release of drugs and site-specific targeting ability. From various studies, it is evident that this lipid-based drug delivery system has improved the oral bioavailability of drugs through better intestinal absorption. Thus, NLCs can be considered as a light of hope in the treatment of various chronic diseases including OC for their advantages when administered orally.

\section{Beneficial aspects of dual targeting over single targeting}

Dual targeting is significantly beneficial over single protein targeting, as there are various research studies carried out in this area proving to be effective.

$\mathrm{Ni}$ W et al. worked on 5-fluorouracil and curcumin loaded nanoparticles for enhanced treatment of hepatocellular carcinoma. Their formulation design was based on the fact that multidrug combination therapy along with targeting techniques will result in increased anticancer effects. In clinical applications, these strategies have become significantly important. They concluded that these dual drug-loaded NPs are a promising tool for cellular targeting and synergistic anticancer efficacy. ${ }^{20}$

Wu S et al. explored that dual drug nanohybrids with the specific targeting capability and high drug loading have gained more importance in cancer therapy. Their formulation was composed of 10-hydroxycamptothecine and methotrexate. They designed a green approach that develops emerging drug delivery systems for cancer treatment. ${ }^{21}$

Jose A et al. developed imatinib and tamoxifen-loaded temperature-sensitive liposomes to target breast cancer. They reported that co-delivery of dual or multiple chemotherapeutic agents using nanocarriers enhances antitumor efficacy, which can be adopted as a potential strategy against breast cancer. ${ }^{22}$

Serri C et al. studied combination chemotherapy that employs two or more drugs, which is prone to suppress the inception of multidrug resistance, exploiting the fact that diverse drugs act in different points of the cellular cycle that amplifies cancer cells. Their results proved that gemcitabine (GMC) and quercetin (QCT) combination showed a synergistic effect in the inhibition of pancreatic cancer cells migration. GMC and QCT were loaded into biodegradable NPs based on poly(lactic-co-glycolic acid), which were externally decorated with hyaluronic acid (HA). HA specifically interacts with the CD44 receptor, for which it plays a major role in tumor targeting. The GMC and QCT loaded NPS decorated with HA showed improved cellular uptake and cytotoxicity. ${ }^{23}$

\begin{tabular}{|c|c|c|c|}
\hline \multicolumn{4}{|c|}{ Table 1: Various drug and their targets approved for } \\
OC. \\
\hline $\begin{array}{c}\text { SL } \\
\text { NO. }\end{array}$ & Drugs & Target & Mechanism \\
\hline 1. & Cyclophosphamide & DNA & Alkylating agent \\
\hline 2. & Melphalan & DNA & Alkylating agent \\
\hline 3. & Thiotepa & DNA & Alkylating agent \\
\hline 4. & Gemcitabine & RRM1 & Antimetabolite \\
\hline 5. & Paclitaxel & TUBB & $\begin{array}{c}\text { Tubulin } \\
\text { depolymerization } \\
\text { inhibitor }\end{array}$ \\
\hline 6. & Doxorubicin & TOP2 & $\begin{array}{c}\text { Topoisomerase } \\
\text { inhibitor }\end{array}$ \\
\hline 7. & Cisplatin & DNA & DNA alkylator \\
\hline 8. & Carboplatin & DNA & DNA alkylator \\
\hline 9. & Bevacizumab & VEGFA & $\begin{array}{c}\text { Monoclonal } \\
\text { Antibody }\end{array}$ \\
\hline 10. & Altretamine & DNA & Alkylating agent \\
\hline 11. & Hydroxyurea & RRM2 & Antimetabolite \\
\hline 12. & Topotecan & TOP1 & $\begin{array}{c}\text { Topoisomerase } \\
\text { inhibitor }\end{array}$ \\
\hline 13. & Niraparib & PARP 1, 2 & PARP inhibitor \\
\hline 14. & Olaparib & PARP 1, 2, 3 & PARP inhibitor \\
\hline 15. & Rucaparib & PARP 1, 2, 3 & PARP inhibitor \\
\hline & & & \\
\hline
\end{tabular}

\section{Various targets in $\mathrm{OC}$}

The following table (Table 1) consists of the various FDA approved drugs available for the treatment of OC. The targets of these drugs are mentioned along with their mechanism of inhibition. This data compilation can serve as a tool for the design and selection of dual or multiple targeting strategies for enhanced efficacy against OC cells. ${ }^{24}$

\section{CONCLUSION}

We conclude that NLCs have a potent scope in the area of chemotherapy. They are advantageous over SLNs and other lipid-based conventional drug delivery systems. Various NLCs loaded with drugs used for OC treatment have shown a path for further development in advanced strategies that increase therapeutic efficacy and safety.

\section{ACKNOWLEDGEMENT}

The authors would like to thank the Department of Science and Technology - Fund for Improvement of Science and Technology Infrastructure in Universities and Higher Educational Institutions (DST-FIST), New Delhi for their infrastructure support to our department. 


\section{CONFLICT OF INTEREST}

The authors declare no conflict of interest.

\section{ABBREVIATIONS}

OC: Ovarian cancer; PTX: Paclitaxel; NLC: Nanostructured lipid carriers; SLN: Solid Lipid Nanoparticle; HA: Hyaluronic acid; GMC: Gemcitabine; QCT: Quercetin.

\section{REFERENCES}

1. Cortez AJ, Tudrej P, Kujawa KA, Lisowska KM. Advances in ovarian cancer therapy. Cancer Chemotherapy and Pharmacology: Springer Verlag 2018;81(1):17-38.

2. Momenimovahed Z, Tiznobaik A, Taheri S, Salehiniya H. Ovarian cancer in the world: Epidemiology and risk factors. Int J Womens Health. 2019;11:28799

3. Pantshwa JM, Kondiah PPD, Choonara YE, Marimuthu T, Pillay V. Nanodrug delivery systems for the treatment of ovarian cancer. Cancers. 2020;12(1):213.

4. Khalifa AM, Elsheikh MA, Khalifa AM, Elnaggar YSR. Current strategies for different paclitaxel-loaded Nano-delivery Systems towards therapeutic applications for ovarian carcinoma: A review article. J Control Release. 2019;311(7):125-37. Available from: https://doi.org/10.1016/j. jconrel.2019.08.034

5. Jaiswal P, Gidwani B, Vyas A. Nanostructured lipid carriers and their current application in targeted drug delivery. Artif Cells, Nanomedicine Biotechnol. 2016;44(1):27-40.

6. Bharali DJ, Khalil M, Gurbuz M, Simone TM, Mousa SA. Nanoparticles and cancer therapy: A concise review with emphasis on dendrimers. Int $\mathrm{J}$ Nanomedicine. 2009;4(1):1-7.

7. Müller RH, Radtke M, Wissing SA. Nanostructured lipid matrices for improved microencapsulation of drugs. Int J Pharm. 2002;242(1-2):121-8.

8. Mehnert W, Mäder K. Solid lipid nanoparticles: Production, characterization and applications. Adv Drug Deliv Rev. 2012;64(suppl.):83-101. Available from: http://dx.doi.org/10.1016/j.addr.2012.09.021

9. Haider M, Abdin SM, Kamal L, Orive G. Nanostructured lipid carriers for delivery of chemotherapeutics: A review. Pharmaceutics. 2020;12(3):288.

10. Selvamuthukumar S, Velmurugan R. Nanostructured Lipid Carriers: A potential drug carrier for cancer chemotherapy. Lipids Health Dis. 2012;11(1):1-8.
11. Wang L, Jia E. Ovarian cancer targeted hyaluronic acid-based nanoparticle system for paclitaxel delivery to overcome drug resistance. Drug Deliv. 2016;23(5):1810-7.

12. Mittal P, Vrdhan H, Ajmal G, Bonde G, Kapoor R, Mishra B. Formulation and Characterization of Genistein-loaded Nanostructured Lipid Carriers: Pharmacokinetic, Biodistribution and in vitro Cytotoxicity Studies. Curr Drug Deliv. 2018;16(3):215-25.

13. Bondì ML, Emma MR, Botto C, Augello G, Azzolina A, DiGaudio F, et al. Biocompatible Lipid Nanoparticles as Carriers to Improve Curcumin Efficacy in Ovarian Cancer Treatment. J Agric Food Chem. 2017;65(7):1342-52.

14. Bang KH, Na YG, Huh HW, Hwang SJ, Kim MS, Kim M, et al. The delivery strategy of paclitaxel nanostructured lipid carrier coated with platelet membrane. Cancers. 2019;11(6):807.

15. Pathak K, Raghuvanshi S. Oral Bioavailability: Issues and Solutions via Nanoformulations. Clin Pharmacokinet. 2015;54(4):325-57.

16. Desai PP, Date AA, Patravale VB. Overcoming poor oral bioavailability using nanoparticle formulations-opportunities and limitations. Drug Discov Today Technol. 2012;9(2):e87-95. Available from: http://dx.doi.org/10.1016/j. ddtec.2011.12.001

17. Poonia N, Kharb R, Lather V, Pandita D. Nanostructured lipid carriers: Versatile oral delivery vehicle. Futur Sci OA. 2016;2(3):FSO135.

18. Iqbal MA, Md S, Sahni JK, Baboota S, Dang S, Ali J. Nanostructured lipid carriers system: Recent advances in drug delivery. J Drug Target. 2012;20(10):813-30.

19. Muchow M, Maincent $P$, Müller RH. Lipid nanoparticles with a solid matrix (SLN®, NLC®, LDC®) for oral drug delivery. Drug Dev Ind Pharm. 2008;34(12):1394-405.

20. Ni W, Li Z, Liu Z, Ji Y, Wu L, Sun S, et al. Dual-Targeting Nanoparticles: Code livery of Curcumin and 5-Fluorouracil for Synergistic Treatment of Hepatocarcinoma. J Pharm Sci. 2019;108(3):1284-95. Available from: https:// doi.org/10.1016/j.xphs.2018.10.042

21. Wu S, Yang X, Lu Y, Fan Z, Li Y, Jiang Y, et al. A green approach to dual-drug nanoformulations with targeting and synergistic effects for cancer therapy. Drug Deliv. 2017;24(1):51-60.

22. Jose A, Ninave KM, Karnam S, Venuganti VVK. Temperature-sensitive liposomes for co-delivery of tamoxifen and imatinib for synergistic breast cancer treatment. Journal of Liposome Research. Taylor and Francis. 2019;29(2):153-62. Available from: http://dx.doi.org/10.1080/08982104.2018 .1502315

23. Serri C, Quagliariello V, laffaioli RV, Fusco S, Botti G, Mayol L, et al. Combination therapy for the treatment of pancreatic cancer through hyaluronic acid-decorated nanoparticles loaded with quercetin and gemcitabine: A preliminary in vitro study. J Cell Physiol. 2019;234(4):4959-69.

24. Markman M. New, Expanded and Modified use of Approved Antineoplastic Agents in Ovarian Cancer. Oncologist. 2007;12(2):186-90.

\section{PICTORIAL ABSTRACT}

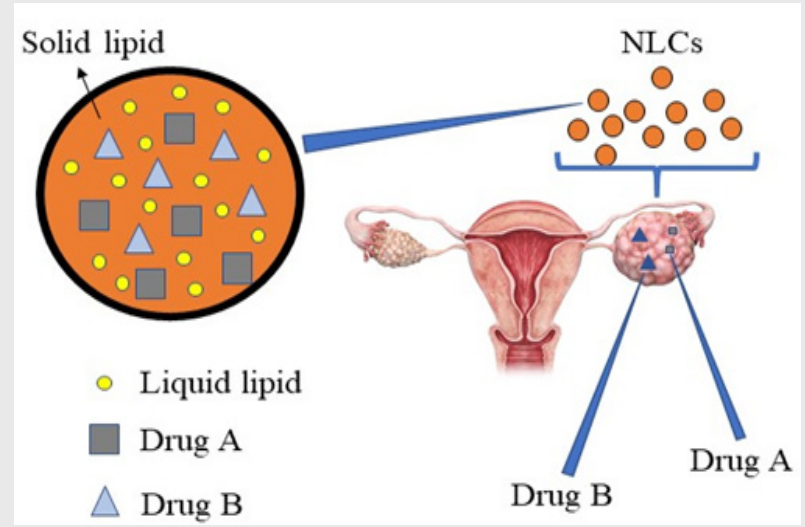

\section{SUMMARY}

One such strategy is oral administration of anticancer agents via NLCs, through which better absorption of drugs and their sustained release can be achieved. Besides this, there is a lack of attention given to multiple proteins targeting in various formulations developed against OC. Those formulations that have dual drug combinations, that is, targeting more than one protein involved in OC pathophysiology as mentioned above have declared better results. Also, various other targets available for ovarian cancer and drugs targeting these were summarised. Further steps include the development of formulation in light of the proposed strategy, in vitro and in vivo characterization to demonstrate the efficacy of the formulation.

Dual targeting approach for ovarian cancer 


\section{About Authors}

Praharsh Kumar M R: He is currently working as a research scholar under the guidance of Dr. N Jawahar in JSS College of Pharmacy, Ooty. He completed Master of Pharmacy from Government College of Pharmacy, Bangalore. He earned B.Pharm in Visveswarapura Institute of Pharmaceutical Sciences.

Dr. N. Jawahar: He earned B.Pharm in AK College of Pharmacy (1999), M. Pharm in KM College of Pharmacy (2002) and PhD from JSS University (2013), Mysore. His experience includes both teaching and research, which is 18.6 years. He has published 81 articles \& presented 30 research papers at national and international levels. Current area of research is Formulation and in vitro/in vivo evaluation of Nano drug delivery systems for improving the bioavailability of poorly soluble drugs and targeting of drugs at specific site.

Cite this article: Kumar PMR, Jawahar N, Raman R, Shivkumar HN, Anjali PB. An Overview of Dual Targteting Nanostructured Lipid Carriers for the Treatment of Ovarian Cancer. Indian $\mathrm{J}$ of Pharmaceutical Education and Research. 2021;55(2):330-5. 